\title{
SYSTEM FORCES IN THE MATHEMATICAL MODEL OF YARN UNWINDING
}

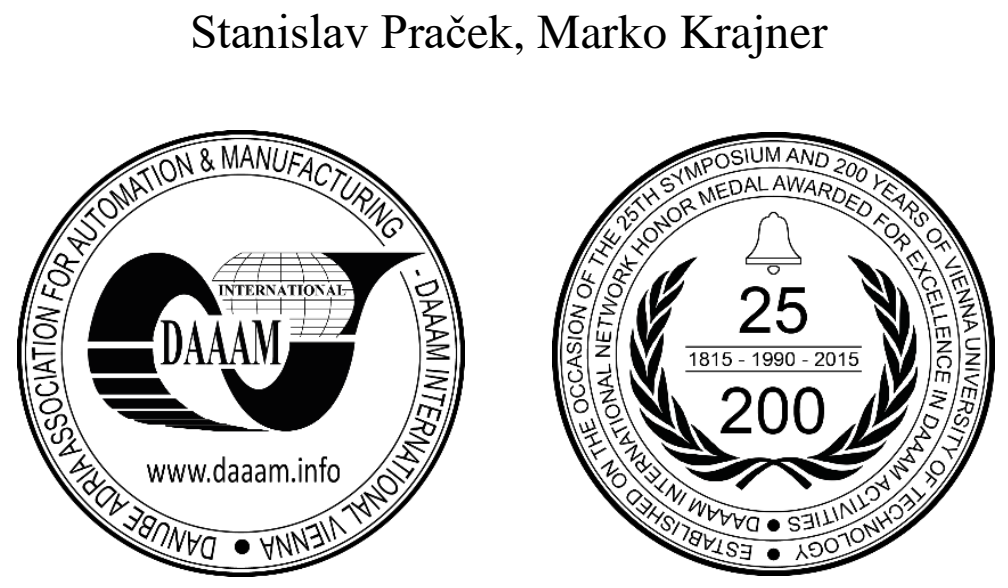

This Publication has to be referred as: Pracek, S[tanislav] \& Krajner, M[arko] (2018). System Forces in the Mathematical Model of Yarn Unwinding, Proceedings of the 29th DAAAM International Symposium, pp.0347-0351, B. Katalinic (Ed.), Published by DAAAM International, ISBN 978-3-902734-20-4, ISSN 1726-9679, Vienna, Austria DOI: $10.2507 / 29$ th.daaam.proceedings.050

\begin{abstract}
The origin of the system forces in equation of motion for yarn is discussed in this paper. We are presented the rotating reference frame is non-inertial and the equation of motion therefore contains three kinds of system forces. In this paper we show how these forces enter the equation of motion for yarn in a rotating reference frame. In addition to the Coriolis and the centrifugal force, there is another fictional force in rotating frames with the dependent angular velocity. We will describe the properties of this force and its effects on dynamics of unwinding yarn.
\end{abstract}

Keywords: dynamics of yarn; balloon theory; non-inertial systems; system forces

\section{Introduction}

The teory of unwinding and balloon formation originate from the pioneering work of D. Padfield[1], [2]. She modified Mack's equations[3] . and included terms that describe the Corolis force. She found solution for a balloon that forms during unwinding from stationary cylindrical package when quasistationary conditions apply. This theory was also used for calculations of multiple balloons and for balloons formed during unwinding from packages with different geometry, such as conic packages[4]. Kothari and Leaf have rederived similar equations, additionally taking into account the effect of gravity and tangential air drag[5],[6]. Using elaborate numerical calculates they have shown that these two forces have only a small contribution to overall dynamics of the yarn. Fraser et al. have applied mathematical theory of perturbations to correctly eliminate the time dependance from equations of motion in stationary conditions[7],[8].Bulding on this foundation we have simplified the problem even further. In papers [9],[10],[11],[12] we have shown the equations of motion in a non-inertial reference frame.

Mathematical modeling is an important tool in determining the optimal yarn motion in textile processes. Simulations make it possible to predict the dynamic properties of the yarn and the mechanical tension inside the yarn, thus it is very valuable technique in the construction of yarn packages and the textile processing equipment. The first step in modeling is to devise a mathematical formulation of the problem. Using suitable assumptions and approximations, one tries to derive the equation of motion of the yarn that is a good description of all the relevant physical effects, but at the same time the equation must be simple enough so that it can be solved at least approximately. 


\section{Non-inertial reference frames and the fictitious forces}

The Newton's second law $\mathbf{F}=$ ma, where $\mathbf{F}$ is the sum of all forces acting on the body, $m$ the mass of the body, and $\mathbf{a}$ its acceleration, takes this form only in inertial reference frames. Inertial references frames are all those reference frames which are not accelerating [1]. In such frames a free body $(\mathbf{F}=0)$ moves with a velocity which is constant both in its direction and its size, since the acceleration a of the body is equal to zero. This statement is also know as the law of inertial motion [2].

If we introduce another reference frame which moves with a constant velocity $\mathbf{V}$ with respect to the inertial reference frame (so that $\mathbf{x}^{\prime}=\mathbf{x}+\mathbf{V t}$ ), then the law of free motion will take the same form as in the first frame, since the acceleration is equal to zero also in the second, $\mathbf{a}^{\prime}=0$ : in case of free motion, the velocity is constant. The new reference system is also inertial. Empirically it is known that the laws of motion are the same in both reference systems; indeed, the two systems are fully equivalent as far as all their mechanical properties are concerned. There is thus an infinity of inertial reference frames which move with constant velocities relative to each other. In all of these systems the laws of mechanics are exactly the same [2]. This is Galileo's principle of relativity, one of the foundations of mechanics.

Now let us consider a reference frame which moves with a relative acceleration $\mathbf{a}_{\text {rel }}$ with respect to a chosen inertial reference frame. In other words, the relation between the acceleration in the inertial frame, a, and the acceleration in the non-inertial frame, $\mathbf{a}^{\prime}$, is given by

$$
\mathbf{a}=\mathbf{a}^{\prime}+\mathbf{a}_{\mathrm{rel}}
$$

If this expression is used in the Newton's second law, we obtain $F=m\left(a^{\prime}+a_{\text {rel }}\right)$. The second term on the right-hand side can be shifted to the left, giving

$$
\mathbf{F}=-\mathrm{ma}_{\mathrm{rel}}=\mathrm{ma}
$$

This equation can be called the Newton's law for a non-inertial reference frame. It shows that in a non-inertial frame the body is accelerated not only because of real external forces which are applied on it, but also because of the fictitious forces $-\mathrm{ma}_{\text {rel }}$. The fictitious forces are not real forces but rather a mathematical trick which simplifies the analysis of mechanical systems, since the equation (2) has exactly the same structure as the standard Newton's law $\mathrm{F}=\mathrm{ma}$ : the acceleration times the mass, ma', is equal to the sum of all external forces $\mathrm{F}$ and all fictitious forces -ma $\mathrm{a}_{\text {rel }}$ that are applied to the body. For this reason, one can make use of all the tools of analytical mechanics if in the equations one replaces the sum of all external forces with the sum of all external and fictitious forces.

The system forces are also known as virtual, inertial or pseudo forces. These names emphasize that these are not real physical forces, but rather effective forces experiences by an observer in an accelerated reference frame due to inertial effects. It must be constantly kept in mind that the fictitious forces do not appear in the equations of motion if these are used to describe motion in an inertial reference frame, even if the motion of the body itself is accelerated. System forces only appear in the equations where the equations themselves are formulated in an accelerated reference frame.

There are numerous situations where the use of non-inertial reference frames is more convenient that that of inertial frames, in spite of the addition of the system forces. In weather prediction it is, for example, necessary to solve a complex system of differential equations in a reference frame which is fixed to the Earth. Since the Earth is not an inertial reference frame (due to its rotation), it is crucial to take into account the Coriolis and centrifugal forces. Particularly important is the Coriolis force which makes the low-pressure systems spin in the counter-clockwise direction in the Northern hemisphere. Non-inertial reference frames are also useful in studying the yarn unwinding. It may be used, for example, when working within the quasi-stationary approximation to describe the yarn unwinding from packages where layers have a large number of yarn loops. On the other hand, when the yarn is being unwound from packages with a small number of loops, the angular velocity of the yarn unwinding is not well defined (constant), thus the problem may just as well be described within a fixed inertial reference frame.

\section{System forces in rotating reference frames with non-constant angular velocity}

Let there be an inertial reference frame $K$ and a non-inertial reference frame $K^{\prime}$, which rotates with respect to the frame $\mathrm{K}$ with an angular velocity $\omega(t)$. The angular velocity is not necessarily constant, thus we are dealing with angular acceleration and deceleration. We consider the motion of a body in both frames. Let the velocity in the frame $\mathrm{K}$ be denoted as v, and the velocity in the frame $K^{\prime}$ as $\mathbf{v}^{\prime}$. Within the time $\delta \mathrm{t}$, the body moves in the frame $K$ by $\mathbf{v} \delta \mathrm{t}$, which must equal $\mathbf{v}^{\prime} \delta \mathrm{t}$ (the displacement of the body in frame $K^{\prime}$ ) and $(\omega(t) \delta t) \times \mathbf{r}^{\prime}$ (the displacement of the body due to the rotation of frame $K^{\prime}$ ). We thus obtain the relation between the two velocities

$$
\mathbf{v}=\mathbf{v}^{\prime}+\omega \times \mathbf{r}^{\prime}
$$

We can also observe that the relation between the time derivative operations with the two coordinate frames may be written as [11] 


$$
\left(\frac{\partial}{\partial t}\right)_{K}=\left(\frac{\partial}{\partial t}\right)_{K^{\prime}}+\omega \times
$$

The operator expression (2) becomes meaningful only when applied to some (vectorial) function p. It is necessary to write $\mathbf{p}$ to the right of each term, obtaining

$$
\left(\frac{\partial \mathbf{p}}{\partial t}\right)_{K}=\left(\frac{\partial \mathbf{p}}{\partial t}\right)_{K^{\prime}}+\boldsymbol{\omega} \times \mathbf{p}
$$

If this rule is applied to the velocity equation (3), we obtain the relation between the accelerations in the inertial frame, a, and the acceleration in the non-inertial frame, a' [7],[10],[13],[14],[15],[16],[17]:

$$
\begin{aligned}
\mathbf{a} & =\mathbf{a}^{\prime}+\omega \times \mathbf{v}^{\prime}+\left(\Phi^{\prime} \times \mathbf{r}^{\prime}+\omega \times \mathbf{v}^{\prime}\right)+\omega \times\left(\omega \times \mathbf{r}^{\prime}\right) \\
& =\mathbf{a}^{\prime}+2 \omega \times \mathbf{v}^{\prime}+\omega \times\left(\omega \times \mathbf{r}^{\prime}\right)+\dot{\Phi} \times \mathbf{r}^{\prime}
\end{aligned}
$$

The last three terms represent the relative acceleration. If this expression is inserted in the Newton's second law for noninertial reference frames, (2), we obtain [11]

$$
\mathbf{F}-2 m \boldsymbol{\omega} \times \mathbf{v}^{\prime}-m \boldsymbol{\omega} \times\left(\boldsymbol{\omega} \times \mathbf{r}^{\prime}\right)-m \dot{\Phi} \times \mathbf{r}^{\prime}=m \mathbf{a}^{\prime}
$$

In a non-inertial reference frame, the body is accelerated not only by the true external forces $\mathbf{F}$, but also by the system forces. These are the Coriolis, centrifugal and Euler's forces.

\section{The equation of motion for yarn}

Assuming negligibly small yarn cross-section and ideal elasticity one may derive the following equation of motion in an inertial reference frame [7],[10],[17]:

$$
\rho D^{2} \mathbf{r}=\frac{\partial}{\partial s}(\mathrm{Tt})+\mathbf{f}
$$

Here $\rho$ is the linear yarn mass density, i.e., the mass of the yarn per unit length which is not necessarily constant in an extensible yarn. The vector $\mathbf{r}(\mathrm{s}, \mathrm{t})$ is the radius vector pointing to an infinitesimal section of the yarn which at time $t$ is locate at the arc-length s away from the origin. The mechanical tension is denoted by $\mathrm{T}(\mathrm{s}, \mathrm{t})$, and $\mathbf{t}(\mathrm{s}, \mathrm{t})$ is the tangential vector to the yarn at the given point; it equals the partial derivative of the radius vector $\mathbf{r}$ with respect to the arc-length $\mathrm{s}$, that is $\mathbf{t}=\partial \mathbf{r} / \partial \mathbf{s}$. The vector $\mathbf{f}$ denotes the linear density of the external forces which are applied to the segment of yarn under discussion. The operator $D$ is the differential operator of the total time derivative following the motion of a point on the yarn.

$$
D=\frac{d}{d t}=\left(\frac{\partial}{\partial t}-V \frac{\partial}{\partial s}\right)
$$

In a rotating reference frame the equation (8) takes a form similar to that of equation (7) [10]:

$$
\rho \mathcal{D}^{2} \mathbf{r}=\frac{\partial}{\partial s}(\mathbf{T t})+\mathbf{f}-2 \rho \boldsymbol{\omega} \times \mathcal{D} \mathbf{r}-\rho \boldsymbol{\omega} \times(\boldsymbol{\omega} \times \mathbf{r})-\rho \dot{\Phi} \times \mathbf{r}
$$

Here $\mathcal{D}$ is the differential operator which follows the motion of a point on the yarn in the rotating reference frame [13]

$$
\mathcal{D}=\left(\left.\frac{\partial}{\partial t}\right|_{K^{\prime}}-V \frac{\partial}{\partial s}\right)
$$

The differential operator $\mathcal{D}$ is related to the operator $\mathrm{D}$ by

$$
D=\mathcal{D}+\omega \times
$$


This expression is similar to the equation (4) here, again, the expression becomes meaningful only when applied to some vectorial function $\mathbf{p}$, so that $\mathrm{D} \mathbf{p}=\mathcal{D} \mathbf{p}+\boldsymbol{\omega}$ xp. The analogy with the Newton's second law for a point particle in a rotating reference frame (1) is manifest. Both equations are similar in their structure and the respective terms may be interpreted in the same way. The density times the acceleration $\mathcal{D}^{2} \mathbf{r}$ is equal to the sum of internal forces $(\partial / \partial \mathrm{s})(\mathrm{Tt})$, external forces $\mathbf{f}$ and the system forces: the Coriolis force, $-2 \rho \boldsymbol{\omega} \times \mathcal{D} \mathbf{r}$ the centrifugal force $-\rho \boldsymbol{\omega} \times(\boldsymbol{\omega} \times \mathbf{r})$ and the force due to angular acceleration $-\rho \varpi \times \mathbf{r}$ (Euler's force).

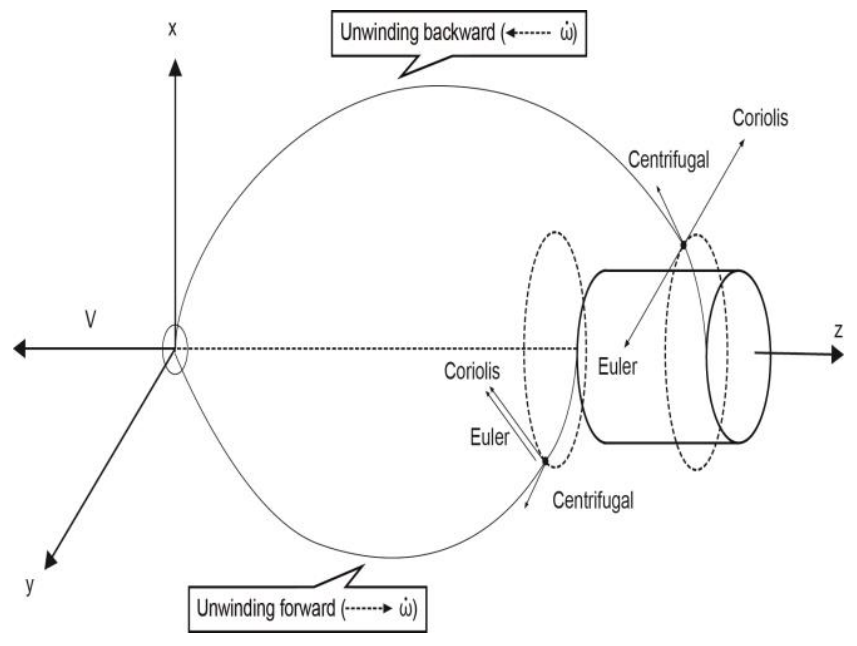

Fig. 1. System forces on a short yarn segment.

The system forces acting on a short yarn segment are shown schematically in Fig. 1. The centrifugal and the Coriolis force are well known, but we would like to emphasize the presence on additional system force $-\rho \ggg \times \mathbf{r}$. This force is oriented along the Coriolis force when the lift-off point is near the front edge of the package. At that moment the angular velocity $\omega$ is increasing [17]. The angular acceleration vector $ळ$ is shown by a dashed line in the figure. When, however, the lift-off point is near the rear edge of the package, the force is oriented in the opposite direction as the Coriolis force, since the angular velocity $\omega$ is decreasing and the angular acceleration $ळ$ points in the opposite direction compared to the situation near the front edge of the package. In the center part of the package the conditions are quasi-stationary (they do change very slowly with time), the additional fictional force is absent. (This is clearly only true for layers with a large number of loops, that is, for precision wound packages.)

\section{The effects of the Euler's force on the yarn dynamics}

The angular velocity $\omega$ is larger during the unwinding in the backward direction as during the unwinding in the forward direction, which is a consequence of a finite winding angle. The difference is very small in densely wound packages, while it may become substantial in cross-wound packages. The importance of the Euler's may be estimated if it is compared with the centrifugal force. The ratio is

$$
\frac{m \dot{r^{\prime}}}{m \omega^{2} r^{\prime}}=\frac{\dot{\Phi}}{\omega^{2}} .
$$

The angular velocity during unwinding in the backward direction is denoted by $\omega_{+}$, while the angular velocity during unwinding in the forward direction is denoted by $\omega_{\text {.. }}$ In the expression above we use the average $\left(\omega_{+}+\omega_{-}\right) / 2$ instead of $\omega_{\text {. }}$ This velocity can be related to the period of the motion (the time during which one loop of yarn is unwound) $\tau_{0}=2 \pi / \omega_{0}$. The angular acceleration can be estimated as

$$
\grave{\Phi}=\frac{\omega_{+}-\omega_{-}}{T}
$$

where $\mathrm{T}$ is the duration of the transition from the unwinding in the forward direction to the backward direction (or vice versa). Since this occurs within the distance of a few loops $n$, we may estimate $T=n \tau_{0}$, where $n$ is small number. We then finally obtain the following expression for the ratio between the two fictional forces:

$$
\frac{\dot{X}}{\omega^{2}}=\frac{n}{2 \pi} \frac{\omega_{+}-\omega_{-}}{\omega_{0}} .
$$


The factor $2 \pi / \mathrm{n}$ may be taken to be approximately equal to 1 , since $\mathrm{n}$ is some small number

$$
\frac{\dot{X}}{\omega^{2}}=\frac{\omega_{+}-\omega_{-}}{\omega_{0}} .
$$

\section{Conclusion}

We have shown the origin of the system forces in the equation of motion in a non-inertial reference frame. These forces are of purely kinematical origin, since they are a consequence of the transition from an inertial to a non-inertial reference frame and they are thereby distinctly different from real ("dynamical") external and internal forces. We have emphasized the commonly neglected Euler's force $-\rho \ggg \times \mathbf{r}$.

The importance of Euler's velocity is equal to the ration between the difference of the angular velocity for unwinding in either direction and their average. This additional system force affects the motion of the yarn at the package edges where the winding angle is reversed. Such a rapid change of the winding angle leads to a sudden change

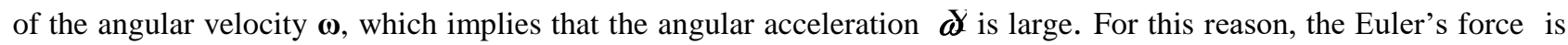
also large and it affects the yarn dynamics. The conditions are then not quasi-stationary but transitory, since the type of motion changes. For this reason there might be instabilities of the balloon shape, the yarn can break. In the future plans, we will measure the tension in yarn during unwinding from packages and our theoretical findings were compared with experimental results.

\section{References}

[1] Padfielf,D.G (1956). A note on fluctuations of tension during unwinding. J.Text.Inst., 47, pp. 301-308.

[2] Padfield, D. G. (1958). The Motion and tension of an unwinding thread. Proc. R. Soc., A 245, pp. $382-407$.

[3] Mack, C. (1953). Theoretical study of ring and cap spinning balloon curves. J.Text.Inst., 44, pp. 483-498.

[4] Booth, H. V. (1957). Variations in tension of an unwinding thread. Br. J. Appl. Phys., 8, pp. 142-144.

[5] Kothari, V. K., Leaf, G. A. V. (1979). The unwinding of yarns from packages. Part I, The theory of yarnunwinding. J. Text. Inst., 3, pp. 89-95.

[6] Kothari, V. K., Leaf, G. A. V. (1979). The unwinding of yarns from packages, Part II:Unwinding from cylindrical packages. J. Text. Inst., 3, pp. 96-104.

[7] Fraser,W. B., Ghosh,T. K., Batra, S. K.(1992). On unwinding yarn from cylindrical package. Proc. R. Soc., A, 436, pp. 479-498.

[8] Fraser WB and Stump DM. (1998). Yarn twist in the ring-spinning balloon. Proc R Soc London, A454, pp. 707723.

[9] Praček S. (2007). Theory of string motion in the textile process of yarn unwinding. International journal of nonlinear sciences and numerical simulation, 8,3 , pp. 451-460.

[10] Praček S, Pušnik N, Franken G, and Simončič B. (2016). Balloon theory of yarn during unwinding from packages. Text. Res. J., 86, 14, pp. 1522-1532.

[11] Praček, S. (2010). Theoretical model of unwinding process from packages, Annals of DAAAM for 2010 \& Proceedings of the 21 st International DAAAM Symposium, 20-23rd October 2010, Zadar, Croatia, ISSN 17269679, ISBN 978-3-901509-73-5, Katalinic, B. (Ed), pp. 1469-1470, Published by DAAAM International Vienna, Vienna

[12] Praček, S. (2010). High-speed yarn transport systems simulation, Annals of DAAAM for 2010 \& Proceedings of the 21 st International DAAAM Symposium, 20-23rd October 2010, Zadar, Croatia, ISSN 1726-9679, ISBN 9783-901509-73-5, Katalinic, B. (Ed), pp. 1471-1472, Published by DAAAM International Vienna, Vienna

[13] Herbert Goldstein, Charles Poole, John Safko. Classical mechanics. 3rd ed. San Francisco;Addison Wesley;Upper Saddle River: Person Education International, cop. 2002

[14] Giancoli, Douglas C. Physics for scientists \& engineers : with modern physics. 4th ed. Upper saddler River: Pearson Education International, cop. 2009

[15] J. A. Roberson in C. T. Crowe. Engineering fluid dynamics. 2th ed. Houghton Mifflin Company, Boston, 1980.

[16] Bronshtein I.N. Handbook of mathematics. 5th ed. Berlin: Springer, cop. 2007

[17] Kong, X. M. Steady state unwinding of yarn from cylindrical packages: theory and experiment. Dissertation, Clemson University, (1997). 